\title{
Influência do tipo de telhado na qualidade da água de chuva coletada em comunidades ribeirinhas
}

A região insular de Belém/PA não é assistida pelo sistema público de abastecimento de água, obrigando seus moradores consumirem água inapropriada dos rios ou se deslocarem para a área continental para obterem água potável. Frente esta realidade e considerando os regimes privilegiados de chuvas da região, a Caritas Metropolitana de Belém implantou microssistemas de captação de água pluvial. O objetivo deste trabalho foi avaliar a influência da superfície de captação pluvial (telhado de palha, barro e fibrocimento) na qualidade de água de chuva destinada ao consumo humano em comunidades ribeirinhas de Belém/PA. Em 33 amostras de águas de chuva coletadas em telhados de barro, palha e fibrocimento foram avaliadas as variáveis: cor aparente, turbidez, sólidos totais dissolvidos, condutividade elétrica, dureza total, alcalinidade, $\mathrm{pH}$ e E.coli., os resultados foram avaliados empregando estatística descritiva e comparados com as diretrizes brasileiras de potabilidade. Todas as amostras apresentaram valores de STD e DT de acordo com a legislação nacional, entretanto entre as superfícies de captação avaliadas, o telhado de fibrocimento apresentou os melhores níveis de COR, TRB, STD e CE e melhor nível microbiológico em função de sua emissividade. Todas as amostras apresentaram níveis microbiológicos em desacordo com os parâmetros de potabilidade, sendo necessária a implantação de um sistema de desinfecção para torná-la apta ao consumo humana.

Palavras-chave: Pluviosidade; Água Potável; Emissividade.

\section{Influence of roof type on the quality of rainwater collected in riverside communities}

\begin{abstract}
The island region of Belém/PA is not assisted by the public water supply system, forcing its residents to consume inappropriate water from rivers or movetothe continental area to obtainem drinking water. Faced with this reality and considering the privileged rainfall regimes of the region,Caritas Metropolitana de Belém implemented rainwater harvesting microsystems. The objective of this work was to evaluate the influence of rainfall catchment (straw roof, clay and fiber cement) on the quality of rainwater intended for human consumption in riverside communities of Belém/PA. In 33 rainwater samples collected on clay, straw and fiber cement roofs, the following variables were evaluated: apparent color, turbidity, total dissolved solids, electrical conductivity, total hardness, alkalinity, $\mathrm{pH}$ and E.coli., the results were evaluated using descriptive statistics and compared with the Brazilian potability guidelines. All samples presented Values of TD and DT according to national legislation, however among the capture surfaces evaluated, the fiber cement roof presented the best levels of COR, TRB, STD and CE and better microbiological level as a function of its emissivity. All samples presented microbiological levels in disagreement with potability parameters, requiring the implementation of a disinfection system to make it suitable for human consumption.
\end{abstract}

Keywords: Rainfall; Drinking Water; Emissivity.

Topic: Uso de Recursos Naturais

Reviewed anonymously in the process of blind peer
Received: $14 / 03 / 2020$

Approved: $22 / 04 / 2020$
Karen Albuquerque Dias da Costa (D) Universidade Federal do Pará, Brasil http://lattes.cnpq.br/8493677235656139 http://orcid.org/0000-0002-1363-303x karenquimica123@yahoo.com.br

Fábio Israel Martins Carvalho (iD Universidade Federal Rural da Amazônia, Brasil http://lattes.cnpq.br/8221002637257793 http://orcid.org/0000-0002-8995-2141 fabioimc@yahoo.com.br

Fernanda Di Paula Belo Ferreira Universidade da Amazônia, Brasil http://lattes.cnpq.br/2561091497327329 fernanda.di.paula@hotmail.com

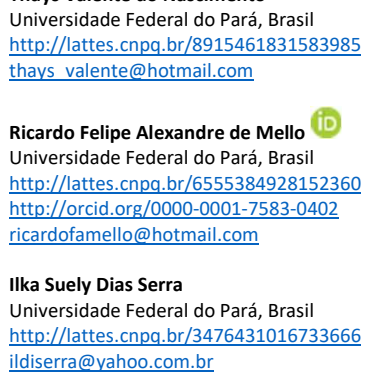

Ilka Suely Dias Serra Universidade Federal do Pará, Brasil http://lattes.cnpq.br/3476431016733666 ildiserra@yahoo.com.br

$\mid \begin{aligned} & \text { Jaqueline Maria Soares da Silva } \\ & \text { Universidade Federal do Pará, Brasil } \\ & \text { http://lattes.cnpq.br/0858733477253042 } \\ & \text { jaquelineifpa@gmail.com } \\ & \text { Maria do Socorro Bezerra Lopes (1) } \\ & \text { Instituto Federal do Pará, Brasil } \\ & \text { http://lattes.cnpq.br/1850698138869190 } \\ & \begin{array}{l}\text { http://orcid.org/0000-0003-1650-4724 } \\ \text { soclopes@gmail.com }\end{array} \\ & \begin{array}{l}\text { Cezarina Maria Nobre Souza } \\ \text { Instituto Federal do Pará, Brasil }\end{array} \\ & \text { http://lattes.cnpq.br/4638345244640854 } \\ & \text { http://orcid.org/0000-0002-2430-4653 } \\ & \text { cezarina.souza@oi.com.br }\end{aligned}$

Kelly das Graças Fernandes Dantas (ID) Universidade Federal do Pará, Brasil http://lattes.cnpq.br/72277777727553334 http://orcid.org/0000-0002-5424-2908 kdgfernandes@ufpa.br

Heronides Adonias Dantas Filho Universidade Federal do Pará, Brasil http://lattes.cnpq.br/7383551694064339 http://orcid.org/0000-0001-5698-5441 herondantas@gmail.com

Referencing this:

COSTA, K. A. D.; CARVALHO, F. I. M.; FERREIRA, F. D. P. B.; NASCIMENTO, T. V.; MELLO, R. F. A.; SERRA, I. S. D.; SILVA, J. M. S.; LOPES, M. S. B.; SOUZA, C. M. N.; DANTAS, K. G. F.; DANTAS FILHO, H. A.. Influência do tipo de telhado na qualidade da água de chuva coletada em comunidades ribeirinhas. Revista Ibero Americana de Ciências Ambientais, v.11, n.3, p.384-391, 2020. DOI: http://doi.org/10.6008/CBPC2179-6858.2020.003.0029 


\section{INTRODUÇÃO}

Nos últimos anos, a falta de água potável é uma ameaça real para inúmeros países (MAHMOOD et al., 2017; NALWANGA et al., 2018; TERÊNCIO et al., 2018). O acelerado aumento populacional, a crescente poluição dos corpos hídricos e as variações climáticas influenciam diretamente na disponibilidade da água no planeta (AL-BATSH et al., 2019; SANTOS et al., 2017). O acesso a água potável é um direito essencial dos indivíduos, fundamental para a manutenção da vida e para o desenvolvimento da humanidade, todavia aproximadamente 2,1 bilhões de pessoas não tem acesso ao abastecimento deste recurso, sendo expostas a doenças como diarreia, cólera e febre tifoide (LAWRIE et al., 2015; MAHMOOD et al., 2017; MUSAYEV et al., 2018; TERÊNCIO et al., 2018). Frente a esta realidade, a captação de água de chuva tem sido investigada, principalmente em regiões de alta pluviosidade, como uma fonte alternativa e economicamente sustentável de abastecimento de água (FARRENY et al., 2011; FUENTES-GALVÁN et al., 2018; LEE et al., 2010; LEE et al., 2012; MAHMOOD et al., 2017; MUSAYEV et al., 2018; QUAGHEBEUR et al., 2019).

A Região Amazônica possui níveis de pluviosidade privilegiados, sendo Belém, capital do Estado do Pará, uma das cidades com os maiores níveis de chuvas (ANANIAS et al., 2010; MOREIRA et al., 2019; NASCIMENTO et al., 2016; SANTOS et al., 2019; TAVARES et al., 2012). Não obstante, as comunidades ribeirinhas residentes na região Insular de Belém não possui abastecimento público de água, obrigando os ribeirinhos deslocarem-se para o continente a fim de obter água potável ou a consumir água inapropriada dos rios (LOBO et al., 2013). Diante desta realidade, a Caritas Metropolitana de Belém (CAMEBE) implantou o projeto: "Água em casa, limpa e saudável”, com o intuito de fornecer água potável através da captação pluvial.

Estudos em vários países incluindo a Palestina, Bangladesh, Índia, Paquistão, Nepal, Malásia Namíbia, Irlanda, EUA, França e Coréia do Sul, tem avaliado a captação pluvial como opção para o abastecimento de água doce (AL-BATSH et al., 2019; ALVES et al., 2014; FUENTES-GALVÁN et al., 2018; LEE et al., 2016; Ll et al., 2010; MENDEZ et al., 2011; QUAGHEBEUR et al., 2019; SANTOS et al., 2017; STURM et al., 2009). Embora seja uma opção ecologicamente atrativa, vários fatores podem influenciar as características desta água, tais como: a poluição atmosférica, o clima local e a superfície de captação (ALVES et al., 2014; LEE et al., 2012; MAHMOOD et al., 2017; MENDEZ et al., 2011; SANTOS et al., 2017; VIALLE et al., 2011). Nesta perspectiva, o objetivo deste trabalho foi avaliar a influência da superfície de captação pluvial na qualidade de água de chuva destinada ao consumo humano em ilhas de Belém-PA.

\section{MATERIAIS E MÉTODOS}

\section{Área de Estudo}

As amostras de água de chuva foram coletadas em diferentes tipos de telhados. As coletas foram realizadas em 5 pontos distribuídos nas ilhas de Jutuba, Ilha Nova e Urubuoca, pertencentes ao município de Belém, no Estado do Pará, onde foi implantado microssistemas de captação pela Caritas Metropolitana de Belém. As coordenadas geográficas dos pontos de coleta são apresentadas na Tabela 1. 
Tabela 1: Localização dos pontos de coleta.

\begin{tabular}{|c|c|c|}
\hline Pontos & Local de amostragem & Coordenadas geográficas \\
\hline 01 & Jutuba & 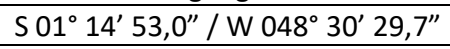 \\
\hline 02 & Jutuba & 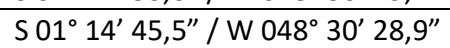 \\
\hline 03 & Jutuba & 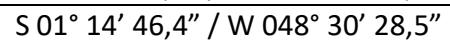 \\
\hline 04 & Urubuoca & 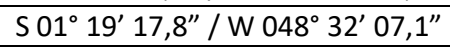 \\
\hline 05 & Ilha Nova & 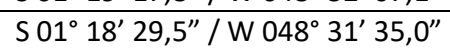 \\
\hline
\end{tabular}

\section{Sistema de captação pluvial implantado}

Os materiais mais frequentes utilizados como coberturas (telhados) das moradas ribeirinhas são: o fibrocimento (TF), a cerâmica vermelha, também chamada de barro (TB) e a palha (TP) (Figura 1).
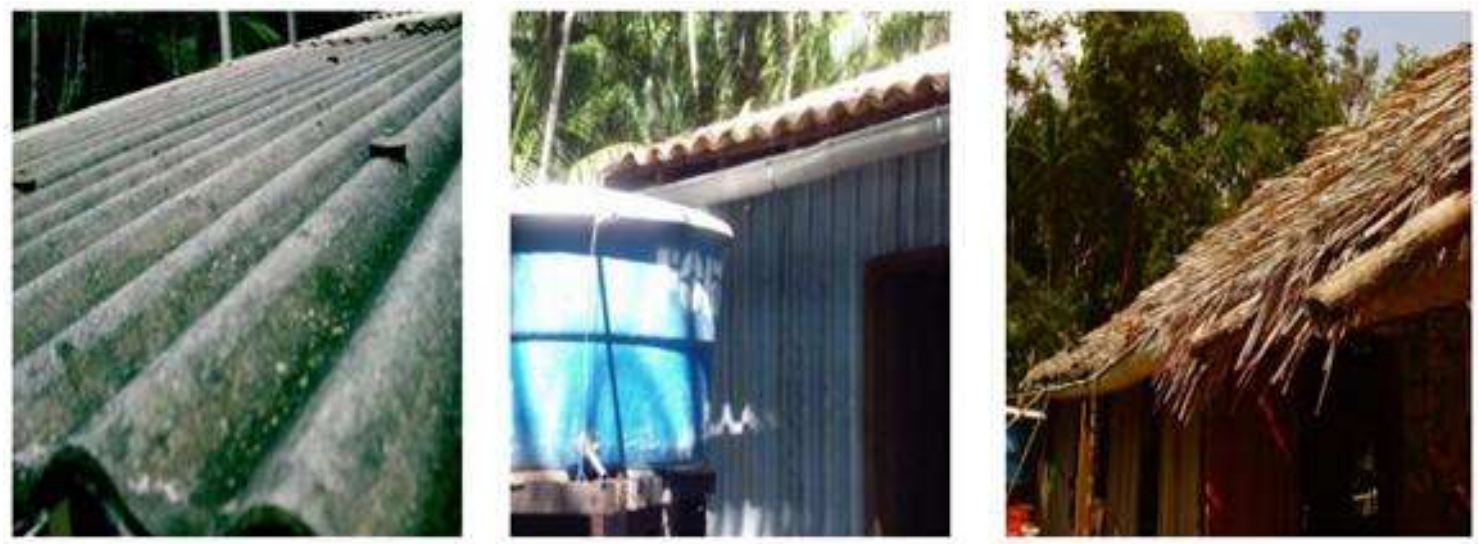

Figura 1: Telhados de fibrocimento, barro e palha.

A água de chuva foi coletada através dos três tipos de superfícies supracitadas e em seguida escoada por meio de calhas de cloreto de polivinila (PVC) para reservatórios de fibra de vidro com capacidade de 1.500 a $2.000 \mathrm{~L}$ suspensos por um suporte de madeira e adaptados uma torneira para a retirada da água armazenada (Figura 2).
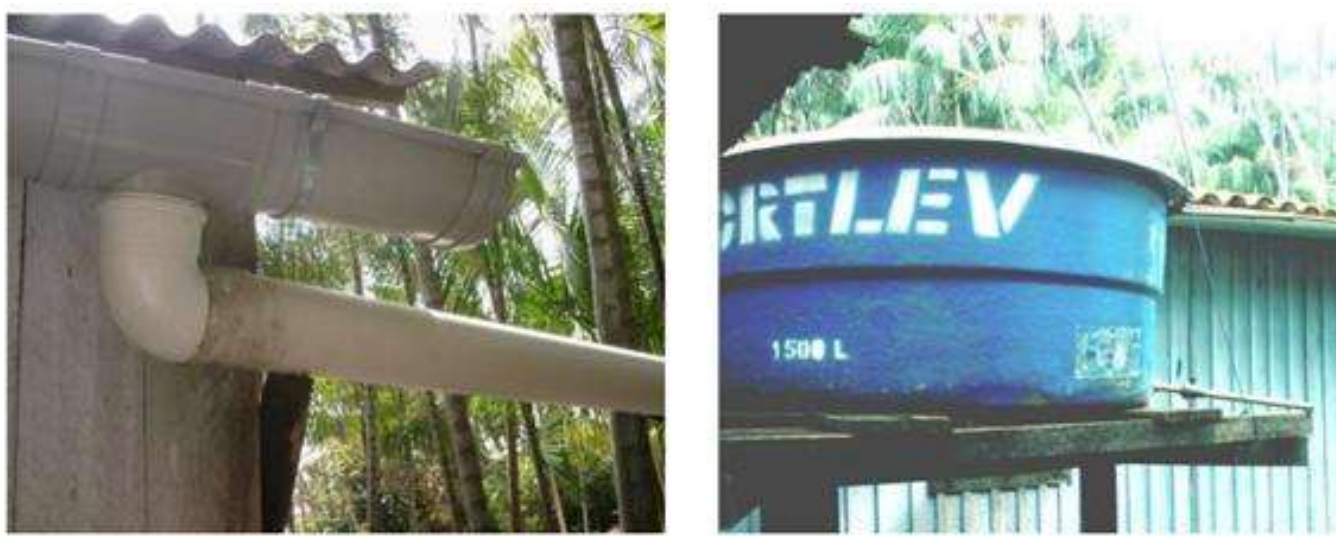

Figura 2: Sistema de captação implantado pela CAMEBE (Caritas Metropolitana de Belém).

\section{Amostragem}

Foram coletadas 33 amostras nos reservatórios que armazenavam a precipitação pluviométrica escoada dos telhados, onde 7 amostras correspondiam à água de chuva captada em TP, 8 em TF e 18 em TB. As amostras foram coletadas em frascos de polietileno $(1.000 \mathrm{~mL})$ e de polipropileno $(200 \mathrm{~mL})$ para a determinação das variáveis físico-químicas e microbiológicas, respectivamente, previamente esterilizadas 
em autoclave a $121^{\circ} \mathrm{C}$, durante 30 minutos. Após a coleta, todas as amostras foram identificadas e em seguida transportadas para o laboratório para a realização das análises físico-químicas e microbiológicas.

\section{Variáveis físico-químicas e microbiológicas}

As variáveis analisadas foram: cor aparente (COR), turbidez (TRB), potencial hidrogeniônico $(\mathrm{pH})$, alcalinidade (ALC), sólidos dissolvidos totais (STD), condutividade elétrica (CE), dureza total (DT) e Escherichia coli (E.coli). Para a determinação da cor aparente se utilizou um espectrofotômetro da Hach, Modelo DR 2500. A turbidez foi quantificada em um Turbidímetro Alfakit, Modelo Microprocessador Plus. Os valores de $\mathrm{pH}$, condutividade elétrica, sólidos totais dissolvidos foram medidos em um aparelho multiparâmetros HANNA Instruments, Modelo HI 255. A alcalinidade e a dureza total foram quantificadas por métodos titulométricos. A variável bacteriológica foi determinada pelo método do substrato cromogênico, com a técnica colillert - Quanti tray - 2000.

Todos os procedimentos de amostragem, armazenamento, preservação e análise das amostras foram realizados conforme as recomendações do manual prático de análise de água e das normas descritas no Standard methods for examination of water and wastewater (APHA et al., 1988; BRASIL, 2006).

\section{Tratamento estatístico dos dados}

O tratamento estatístico dos dados foi realizado através de estatística descritiva, considerando os resultados de média, desvio padrão, mínimo e máximo e percentual de atendimento a legislação brasileira de potabilidade de água. Foram empregados o software pago Microsoft Excel e o software livre BioStat 5.0, desenvolvido pela Universidade Federal do Pará.

\section{RESULTADOS}

\section{Variáveis físico-químicas e microbiológicas da água de chuva coletadas em diferentes telhados}

Os valores médios e os desvios padrão, os percentuais de atendimento as normas de potabilidade das variáveis físico-químicas e microbiológicas para as amostras de água de chuva coletadas nos telhados de barro (TB), de fibrocimento (FB) e telhado de palha (TP) são apresentados na Tabela 2.

Tabela 2: Variáveis físico-químicas e microbiológicas da água de chuva coletadas em diferentes telhados.

\begin{tabular}{|l|l|l|l|l|l|l|l|l|}
\hline Variáveis & Unidades & $\begin{array}{l}\text { Telhado de } \\
\text { Barro }\end{array}$ & Telhado de Fibrocimento & \multicolumn{2}{l|}{$\begin{array}{l}\text { Telhado de } \\
\text { Palha }\end{array}$} & \multicolumn{2}{l|}{$\begin{array}{l}\text { \% de atendimento } \\
\text { nédiocionais }\end{array}$} \\
\cline { 3 - 9 } & & $\begin{array}{l}\text { Média } \pm \\
\text { Desvio Padrão }\end{array}$ & $\begin{array}{l}\text { Média } \pm \\
\text { Desvio Padrão }\end{array}$ & $\begin{array}{l}\text { Média } \pm \\
\text { Desvio Padrão }\end{array}$ & TB & TF & TP \\
\hline COR & UH & $33,89 \pm 27,82$ & $17,35 \pm 12,37$ & $111,85 \pm 50,59$ & 33,3 & 62,5 & 0,0 & 15 \\
\hline TRB & UT & $1,44 \pm 1,99$ & $0,20 \pm 0,29$ & $8,32 \pm 7,12$ & 88,9 & 100 & 57,1 & 5 \\
\hline STD & $\mathrm{mg} / \mathrm{L}$ & $48,02 \pm 110,62$ & $22,59 \pm 7,11$ & $124,67 \pm 230,87$ & 100 & 100 & 100 & 1000 \\
\hline CE & MS.cm -1 & $101,18 \pm 219,45$ & $43,70 \pm 34,77$ & $245,75 \pm 460,64$ & - & - & - & - \\
\hline DT & $\mathrm{mg} / \mathrm{L}$ & $8,88 \pm 12,79$ & $21,35 \pm 15,65$ & $26,83 \pm 22,12$ & 100 & 100 & 100 & 500 \\
\hline pH & - & $6,72 \pm 0,56$ & $7,01 \pm 0,42$ & $6,63 \pm 0,44$ & 88,9 & 100 & 100 & $6,0-9,5$ \\
\hline ALC & $\mathrm{mg} / \mathrm{L}$ & $3,64 \pm 2,88$ & $8,37 \pm 5,75$ & $10,50 \pm 9,97$ & - & - & - & 500 \\
\hline E.coli & $\mathrm{NMP} / 100 \mathrm{~mL}$ & $102,7 \pm 195,27$ & $3,58 \pm 4,38$ & $55,47 \pm 70,92$ & 0 & 0 & 0 & 0 \\
\hline
\end{tabular}


As variáveis físico-químicas cor aparente (COR), a turbidez (TRB), sólidos totais dissolvido (STD) e condutividade elétrica (CE) das amostras de água de chuva foram maiores para o telhado de palha, seguido do telhado de barro e fibrocimento. A COR média das amostras de água de chuva coletadas foram: 111,85 UH (TP), 33,89 UH (TB), 17,25 UH (TF). Embora os valores médios estejam acima de 15 UH, avaliando-se os valores individuais, constatou-se que $62,5 \%$ e $33,3 \%$ das amostras coletadas em telhados de fibrocimento e palha, respectivamente, estão de acordo com a legislação brasileira. A TRB média nas amostras de água de chuva coletadas foram: TP (8,32 UT), TB (1,44 UT) e TF (0,20 UT). O percentual de atendimento a legislação brasileira para TRB foi de 100 \% (TF), 88,9\% (TB) e 57,1\% (TP). Para todas as superfícies de captação, os níveis STD mostraram-se dentro dos limites determinados pela legislação (1.000 mg. L $\left.^{-1}\right)$. O teor médio de STD e de CE nas amostras foi respectivamente: TP $\left(124,67 \mathrm{mg} . \mathrm{L}^{-1} ; 245,75 \mu \mathrm{S} . \mathrm{cm}^{-1}\right)$, TB $\left(48,02 \mathrm{mg} . \mathrm{L}^{-1} ; 101,18 \mu \mathrm{S} . \mathrm{cm}^{-1}\right)$, $\operatorname{TF}\left(22,59 \mathrm{mg} . \mathrm{L}^{-1}\right)$ e $\mathrm{CE}\left(43,70 \mu \mathrm{S} . \mathrm{cm}^{-1}\right)$.

No que se refere à dureza total (DT), todas as amostras apresentaram valores abaixo do limite máximo permitido pela legislação (500 mg. $\mathrm{L}^{-1}$ ), variando de 8,0 a 56,0 mg. $\mathrm{L}^{-1}$ para TP, de 0,0 a $40,0 \mathrm{mg} \cdot \mathrm{L}^{-1}$ para TF e de 0,0 a $44,0 \mathrm{mg} \cdot \mathrm{L}^{-1}$ para TB, com médias iguais a 26,$83 ; 21,35$ e $8,88 \mathrm{mg} \cdot \mathrm{L}^{-1}$, respectivamente. Para todas as superfícies de captação as amostras foram classificadas como águas moles, ou seja, DT inferior a 50 mg. $\mathrm{L}^{-1}$. Os valores médios de pH foram 6,63 (TP), 6,72 (TB) e 7,01 (TF) e os de ALC foram de TP 10,5 mg. L $^{-1}$ ( $T P)$, seguido pelo TF (8,37 mg. $\left.\mathrm{L}^{-1}\right)$ e TB $\left(3,64 \mathrm{mg} \cdot \mathrm{L}^{-1}\right)$, estando dentro dos limites estabelecidos no Brasil.

Para a avaliação da qualidade da água de chuva como fonte de água potável, quantificou-se E.coli como indicador microbiológico. A legislação brasileira define que o número E.coli por $100 \mathrm{~mL}$ de água deve ser igual à zero, todavia todas as superfícies de captação apresentaram amostras com níveis inadequados de CT ao consumo humano. O valor médio de E.coli foi: 102,70 NMP.100 mL $\mathrm{L}^{-1}$ para TB, 55,47 NMP.100 mL ${ }^{-1}$ para TP e 3,58 NMP.100 $\mathrm{mL}^{-1}$ para TF.

\section{DISCUSSÃO}

Neste trabalho foi avaliada a influência dos tipos de telhados (palha, barro e fibrocimento) na qualidade da água de chuva coletada para abastecimento humano de comunidades ribeirinhas de Belém-PA. A captação de água de chuva em telhados é uma alternativa promissora para o abastecimento de água doce para regiões de alta pluviosidade, poluição atmosférica e assoladas pela escassez (FARRENY et al., 2011; MENDEZ et al., 2011; ZDEB et al., 2020). Estima-se que a captação da água de chuva pode ofertar de 12 a 100\% do volume de água necessário para a manutenção de uma família (MUSAYEV et al., 2018). As águas de chuva são consideradas águas limpas e de boa qualidade para aproveitamento doméstico, todavia estas podem ser contaminadas por impurezas e microrganismos presentes nas superfícies de captação, bem como através da degradação dos materiais constituintes dos telhados (LEONG et al., 2017; MAHMOOD et al., 2017; NOSRATI, 2017; SANTOS, et al., 2017).

Comparando-se as superfícies de captação, observa-se que as amostras escoadas em telhado de fibrocimento apresentam os menores valores de COR, TRB, STD e CE, seguida das amostras coletadas em 
telhado de barro e palha. $O$ telhado de palha, cobertura comum de casas ribeirinhas de regiões mais isoladas, apresenta alta rugosidade, fator que contribui para o acúmulo de poluentes do ar, resíduos da erosão do solo depositados pelo vento e detritos de animais e folhas oriundas da vegetação presente no entorno da área de coleta (LEE et al., 2012; ZDEB et al., 2020; ZHANG et al., 2014). Este tipo de cobertura também é suscetível a sofrer uma contínua deterioração devido ser constituída de matéria orgânica, haja vista que são estrutura é formada primordialmente por folhas secas da palmeira Euterpe oleracea Mart.

Observa-se que os valores de $\mathrm{pH}$ foram próximos a faixa próxima a neutralidade $(\mathrm{pH} 6,0-7,9)$ em todas as amostras. Estudos em outros países obtiveram valores de $\mathrm{pH}$ para água escoada de telhados próximos aos encontrados neste trabalho: 6,10 nos EUA, 6,50 na França, 6,00 - 7,9 na Coreia do Sul (LEE et al., 2012; MENDEZ et al., 2011; VIALLE et al., 2011). Cabe ressaltar, que as amostras de água de chuva coletadas no telhado de fibrocimento apresentaram maiores de valores de $\mathrm{pH}$ que as de outros tipos de telhados. Este fato pode ser associado a composição química de telhados de fibrocimento constituída de espécies de natureza alcalina como: calcário, argila, $\mathrm{Ca}^{+2}, \mathrm{Mg}^{+2}, \mathrm{OH}^{-}, \mathrm{HCO}_{3}{ }^{-}$e $\mathrm{CO}_{3}{ }^{-}$(GIKAS et al., 2012; LEE et al., 2012; MENDEZ et al., 2011; ZDEB et al., 2020; ZHANG et al., 2014). Estas espécies químicas associadas as sujidades depositadas pelo vento nos telhados podem ser responsáveis pelos valores próximos de ALC e DT entre o telhado de palha e de fibrocimento.

A presença de indicadores fecais na água de chuva pode estar relacionada com a lixiviação de excrementos de pássaros e de pequenos mamíferos depositados nos telhados (BAE et al., 2019; LEE et al., 2012; MAHMOOD et al., 2017; NALWANGA et al., 2018; SANTOS, 2017; ZDEB et al., 2020). Dentre os resultados microbiológicos, observou-se que as amostras coletadas em telhado de fibrocimento apresentaram os menores teores de E.coli, este fato pode ser explicado através da baixa emissividade deste material, que quando exposto ao sol sofre a elevação da temperatura superficial inativando frações deste indicador microbiológico (LEE et al., 2012; MENDEZ et al., 2011; ZDEB et al., 2020).

\section{CONCLUSÕES}

As amostras de água de chuva coletadas para o abastecimento humano em comunidades ribeirinhas de Belém-PA apresentaram qualidade físico-química e microbiológica distintas em função dos tipos de telhados. A água de chuva coletada em telhados de fibrocimento apresentou os resultados mais aceitáveis frente a legislação brasileira para COR e os menores valores de TRB, STD e CE. Para o telhado de fibrocimento, também foram encontrados os menores níveis do indicador microbiológico E.coli, resultado associado principalmente a emissividade do fibrocimento. Todas as amostras de água de chuva coletadas, apresentaram valores de E.coli em desacordo com a legislação nacional, sugerindo-se a associação de um sistema de desinfecção para a melhora da qualidade microbiológica da água captada para poder destiná-la para fins potáveis.

AGRADECIMENTOS: ao Conselho Nacional de Desenvolvimento Científico e Tecnológico (CNPQ), pelo 
suporte financeiro desta pesquisa (Edital MCT/CT-SAÚDE/CT-HIDRO/CNPq N 45/2008), pela concessão de bolsa de mestrado a K.A.D.C (CNPq processo: 135151/2010-4), e a Caritas Metropolitana de Belém (CAMEBE) por fornecer os meios de transporte para as visitas em campo.

\section{REFERÊNCIAS}

AL-BATSH, N.; AL-KHATIB, I. A.; GHANNAM, S.; ANAYAH, F.; JODEH, S.; HANDABALI, G.;KHALAF, B.; VAN DER VALK, M.. Assessment of rainwater harvesting systems in poor rural communities: A case study from Yatta Area, Palestine. Water, v.11, n.3, p.1-15, 2019.DOI: https://doi.org/10.3390/w11030585

ALVES, F.; KOCHLIN,T.; LUZ, J.; SANTOS, S. M.; GAVAZZA, S.. Water quality and microbial diversity in cisterns from semiarid areas in Brazil. Journal of Water and Health, v.12, n.3, p.513-525, 2014. DOI: https://doi.org/10.2166/wh.2014.139

ANANIAS, D. S.; SOUZA, E. B.; DOUZA, P. F. S.; SOUZA, A. M. L.; VITORINO, M. I.; TEIXEIRA, G. M.; FERREIRA, D. B. S. Climatologia da estrutura vertical da atmosfera em novembro para Belém/PA. Revista Brasileira de Meteorologia, v.25, n.2, p.218-226, 2010. DOI: https://doi.org/10.1590/S0102-77862010000200006

APHA; AWWA; WEF. American Public Health Association; American Water Works Association; World Economic Forum. Standard methods for the examination of water and wastewater. $20 \mathrm{ed}$. Washington: APHA, 1988.

BAE, S.; MAESTRE, J. P.; KINNEY, K. A.; KIRISITS, M. J.. An examination of the microbial community and occurrence of potential human pathogens in rainwater harvested from different roofing materials. Water Research, v.159, p.406413, 2019. DOI:

https://doi.org/10.1016/j.watres.2019.05.029

BRASIL. Manual Prático de Análise de Água. 4 ed. Brasília: Fundação Nacional de Saúde, 2006.

FARRENY, R.; MORÁLES-PINZÓN, T.; GUISASOLA, A.; TAYÁ, C.; GABARRELL, X.. Roof selection for rainwater harvesting: Quantity and quality assessments in Spain. Water Research, v.45, n.10, p.3245-3254, 2011. DOI: https://doi.org/10.1016/j.watres.2011.03.036

FUENTES-GALVÁN, M. L.; MEDEL, J. O.; HERNÁNDEZ, L. A.. Roof rainwater harvesting in central Mexico: Uses, benefits, and factors of adoption. Water, v.10, n.2, 2018. DOI: https://doi.org/10.3390/w10020116

GIKAS, G. D.; TSIHRINTZIS, V. A.. Assessment of water quality of first-flush roof runoff and harvested rainwater. Journal of Hydrology, v.466-467, p.115-126, 2012. DOI: http://dx.doi.org/10.1016/i.jhydrol.2012.08.020

LAWRIE, K.; MILLS, A.; FIGUEREDO-FERNÁNDEZ, M.; GUTIERREZ-ALFARO, S.; MANZANO, M.; SALADIN, M.. UV dosimetry for solar water disinfection (SODIS) carried out in different plastic bottles and bags. Sensors and Actuators, B: Chemical, v.208, p.608-615, 2015. DOI:

http://dx.doi.org/10.1016/i.snb.2014.11.031
LEE, J.; YANG, J. S.; HAN, M.; CHOI, J. U.. Comparison of the microbiological and chemical characterization of harvested rainwater and reservoir water as alternative water resources. Science of the Total Environment, v.408, n.4, p.896-905, 2010. DOI: http://dx.doi.org/10.1016/i.scitotenv.2009.11.001

LEE, J. Y.; BAK, G.; HAN, M.. Quality of roof-harvested rainwater: Comparison of different roofing materials. Environmental Pollution, v.162, p.422-429, 2012. DOI: http://dx.doi.org/10.1016/j.envpol.2011.12.005

LEE, K. E.; MOKHTAR, M.; HANAFIAH, M. M.; HALIM, A. A.; BADUSAH, J.. Rainwater harvesting as an alternative water resource in Malaysia: Potential, policies and development. Journal of Cleaner Production, v.126, p.218-222, 2016. DOI: https://doi.org/10.1016/j.jclepro.2016.03.060

LEONG, J. Y. C.; CHONG, M. N.; POH, P. E.; HERMAWAN, A.; TELEI, A.. Longitudinal assessment of rainwater quality under tropical climatic conditions in enabling effective rainwater harvesting and reuse schemes. Journal of Cleaner

Production, v.143, p.64-75, 2017. DOI:

http://dx.doi.org/10.1016/j.jclepro.2016.12.149

LI, Z.; BOYLE, F.; REYNOLDS, A.. Rainwater harvesting and greywater treatment systems for domestic application in Ireland. Desalination, v.260, n.1-3, p.1-8, 2010. DOI: http://dx.doi.org/10.1016/i.desal.2010.05.035

LOBO, M. A. A.; LIMA, D. M. B.; SOUZA, C. M. N.; NASCIMENTO, W. A.; ARAÚJO, L. C. C.; SANTOS, N. B.. Avaliação econômica de tecnologias sociais aplicadas à promoção de saúde: Abastecimento de água por sistema Sodis em comunidades ribeirinhas da Amazônia. Ciência e Saude Coletiva, v.18, n.7, p.2119-2127, 2013. DOI: https://doi.org/10.1590/S1413-81232013000700027

MAHMOOD, A.; HOSSAIN, F.. Feasibility of managed domestic rainwater harvesting in South Asian rural areas using remote sensing. Resources, Conservation and Recycling, v.125, n.2016, p.157-168, 2017. DOI: http://dx.doi.org/10.1016/j.resconrec.2017.06.013

MENDEZ, C. B.; KLENZENDORF, J. B.; AFSHAR, B. R.; SIMMONS, M. T.; BERRETT, M. E.; KINNEY, K. A.; KIRISITS, M. J.. The effect of roofing material on the quality of harvested rainwater. Water Research, v.45, n.5, p.2049-2059, 2011. DOI: http://dx.doi.org/10.1016/i.watres.2010.12.015

MOREIRA, F. S. A.; FERREIRA, G. R. B.; DIAS, L. C.; VITORINO, M. I.. Variabilidade da precipitação na Cidade de Belém/PA e sua relação com a incidência de Leptospirose. Revista Brasileira de Geografia Física, v.12, n.1, p.71-80, 2019.

MUSAYEV, S.; BURGESS, E.; MELLOR, J.. A global 
performance assessment of rainwater harvesting under climate change. Resources, Conservation and Recycling, v.132, n.1, p.62-70, 2018. DOI:

https://doi.org/10.1016/i.resconrec.2018.01.023

NALWANGA, R.; MUYANJA, C. K.; MCGUIGAN, K. G.; QUITY, B.. A study of the bacteriological quality of roof-harvested rainwater and an evaluation of SODIS as a suitable treatment technology in rural Sub-Saharan Africa. Journal of Environmental Chemical Engineering, v.6, n.3, p.3648-3655, 2018. DOI: http://dx.doi.org/10.1016/j.jece.2016.12.008

NASCIMENTO, T. V.; FERNANDES, L. L.; YOSHINO, G. H.. Potencial de aproveitamento de água de chuva na Universidade Federal do Pará. Revista Monografias Ambientais, v.15, n.1, p.105-116, 2016. DOI: http://dx.doi.org/10.5902/223613085341

NOSRATI, K.. Identification of a water quality indicator for urban roof runoff. Sustainability of Water Quality and Ecology, v.9-10, p.78-87, 2017. DOI: https://doi.org/10.1016/j.swaqe.2017.07.001

QUAGHEBEUR, W.; MULHERN, R. E.; RONSSE, S.; HEYLEN, S.; BLOMMAERT, H.; POTEMANS, S.; MENDIZÁBAL, C. V.; GÁRCIA, J. T.. Arsenic contamination in rainwater harvesting tanks around Lake Poopó in Oruro, Bolivia: An unrecognized health risk. Science of the Total Environment, v.688, p.224230, 2019. DOI:

https://doi.org/10.1016/j.scitotenv.2019.06.126

SANTOS, S. M.; FARIAS, M. M. M. W. E. C.. Potential for rainwater harvesting in a dry climate: Assessments in a semiarid region in northeast Brazil. Journal of Cleaner Production, v.164, p.1007-1015, 2017. DOI: http://dx.doi.org/10.1016/i.jclepro.2017.06.251

SANTOS, M. R. S.; VITORINO, M. I.; SODRÉ, G. R. C.; OLIVEIRA, V. G.. Identificação de extremos de chuva na mesorregião metropolitana de Belém: o uso do modelo box plot para a gestão socioambiental. Revista Brasileira de Geografia Física, v.12, n.4, p.1554-1562, 2019.

STURM, M.; ZIMMERMANN, M.; SCHUTZ, K.; URBAN, W.; HARTUNG, H.. Rainwater harvesting as an alternative water resource in rural sites in central northern Namibia. Physics and Chemistry of the Earth, v.34, n.13-16, p.776-785, 2009. DOI: http://dx.doi.org/10.1016/j.pce.2009.07.004

TAVARES, J. P. N.; MOTA, M. A. S.. Condições termodinâmicas de eventos de precipitação extrema em Belém-PA durante a estação chuvosa. Revista Brasileira de Meteorologia, v.27, n.2, p.207-218, 2012. DOI: https://doi.org/10.1590/S0102-77862012000200007

TERÊNCIO, D. P. S.; FERNANDES, L. F. S.; CORTES, R. M. V.; MOURA, R. M. V.; PACHECO, F. A. L.. Rainwater harvesting in catchments for agro-forestry uses: A study focused on the balance between sustainability values and storage capacity. Science of the Total Environment, v.613-614, p.1079-1092, 2018. DOI: https://doi.org/10.1016/j.scitotenv.2017.09.198

VIALLE, C.; SABLAYROLLES, C.; LOVERA, M.; JACOB, S.; HUAU, M. C.; MONTREJAUD-VIGNOLES, M.. Monitoring of water quality from roof runoff: Interpretation using multivariate analysis. Water Research, v.45, n.12, p.3765-3775, 2011. DOI: http://dx.doi.org/10.1016/j.watres.2011.04.029

ZDEB, M.; ZOMORSKA, J.; PAPCIAK, D.; SLYS, D.. The quality of rainwater collected from roofs and the possibility of its economic use. Resources, v.9, n.2, 2020. DOI: https://doi.org/10.3390/resources9020012

ZHANG, Q.; WANG, X.; HOU, P.; WAN, W.; LI, R.; REN, Y. R.; OUYAHNG, Z.. Quality and seasonal variation of rainwater harvested from concrete, asphalt, ceramic tile and green roofs in Chongqing, China. Journal of Environmental Management, v.132, p.178-187, 2014. DOI: http://dx.doi.org/10.1016/j.jenvman.2013.11.009

A CBPC - Companhia Brasileira de Produção Científica (CNPJ: 11.221.422/0001-03) detém os direitos materiais desta publicação. Os direitos referem-se à publicação do trabalho em qualquer parte do mundo, incluindo os direitos às renovações, expansões e disseminações da contribuição, bem como outros direitos subsidiários. Todos os trabalhos publicados eletronicamente poderão posteriormente ser publicados em coletâneas impressas sob coordenação da Sustenere Publishing, da Companhia Brasileira de Produção Científica e seus parceiros autorizados. Os (as) autores (as) preservam os direitos autorais, mas não têm permissão para a publicação da contribuição em outro meio, impresso ou digital, em português ou em tradução. 